\title{
AGN with extreme X-ray amplitude variations
}

\author{
D. Grupe ${ }^{1, a}$, S. Komossa ${ }^{2}$, K.M. Leighly ${ }^{3}$, and L.C. Gallo ${ }^{4}$ \\ ${ }^{1}$ Department of Astronomy \& Astrophysics, Pennsylvania State University, 525 Davey Lab, University Park, \\ PA 16802, USA \\ ${ }^{2}$ Max-Planck-Institut für Radioastronomie, Auf dem Hügel 69, 53121 Bonn, Germany \\ ${ }^{3}$ Homer L. Dodge Department of Physics and Astronomy, University of Oklahoma, 440 W. Brooks St., Norman, \\ OK 73019, USA \\ ${ }^{4}$ Department of Astronomy and Physics, Saint Mary's University, Halifax, NS B3H 3C3, Canada
}

\begin{abstract}
We present active galactic nuclei (AGNs) that have shown extreme amplitude variability in X-rays. These AGN appear as bright for long periods, but then suddenly become extremely X-ray weak sources. Most likely this behavior is due to strong absorption along the line of sight or by relativistically blurred reflection. Two extreme examples are the Narrow Line Seyfert 1 (NLS1) galaxies WPVS 007 and Mkn 335. WPVS 007 is a peculiar AGN because it combines the properties of a normal NLS1 with those of a broad-absorption line (BAL) quasar, which typically only appear in high-luminousity, high black hole mass systems. Mkn 335 has appeared an an X-ray bright AGN for most of the past few decades, but was caught by Swift in a deep X-ray flux minimum state in 2007, and has remained in that state for most of the time since. One potential explanation for this low state is absorption. Several Other AGN have been in deep minimum X-ray flux states, including PG 0844+349 and $1 \mathrm{H}$ 0707-495, for which the dramatic drops in X-ray flux have been explained by blurred X-ray reflection.
\end{abstract}

\section{INTRODUCTION}

The highest amplitudes of X-ray variability ever observed (factors >1000-6000), have been from otherwise inactive galaxies, and these represent the best candidates to date for stellar tidal disruption events (TDEs). AGN typically vary by factors of a few in X-rays, while amplitudes of variability exceeding a factor of $70-100$ are extremely exceptional. In this contribution, we focus on highamplitude variability in classical AGN and consider the possible causes.

A successful way to discover X-ray variability is through X-ray surveys, like the ROSAT All-Sky Survey (RASS). Figure 1 displays the RASS and pointed ROSAT PSPC count rates of the sample of bright soft X-ray selected AGN [17]. In addition to IC 3599 [2,14,27], there are three more sources in that sample which display dramatic decreases in their ROSAT PSPC counts rates: WPVS 007, RX J2217.9-5941, and RX J1624.9+7554 $([15,18]$, and [16], respectively). The latter is an inactive galaxy and our interpretation of the X-ray outburst in this source was a TDE, similar to other ROSAT-discovered sources like NGC 5905 (e.g., [27]). However, the other two AGN are Narrow Line Seyfert 1 galaxies (NLS1s), a class of type-1 AGN that is typically characterized by extreme variability. WPVS 007 appears extreme even amongst this class.

Recently, other NLS1s have been discovered with Swift in extremely low X-ray flux states, for example Mkn 335, 1H0707-495, and PG0844+349. Yet another NLS1, PHL $1092[34,35]$ was caught by XMM-Newton displaying a dramatic drop in its X-ray flux by a factor of 260 (see also G. Miniutti's article in these proceedings).

\footnotetext{
a e-mail: dxg35@psu.edu
}

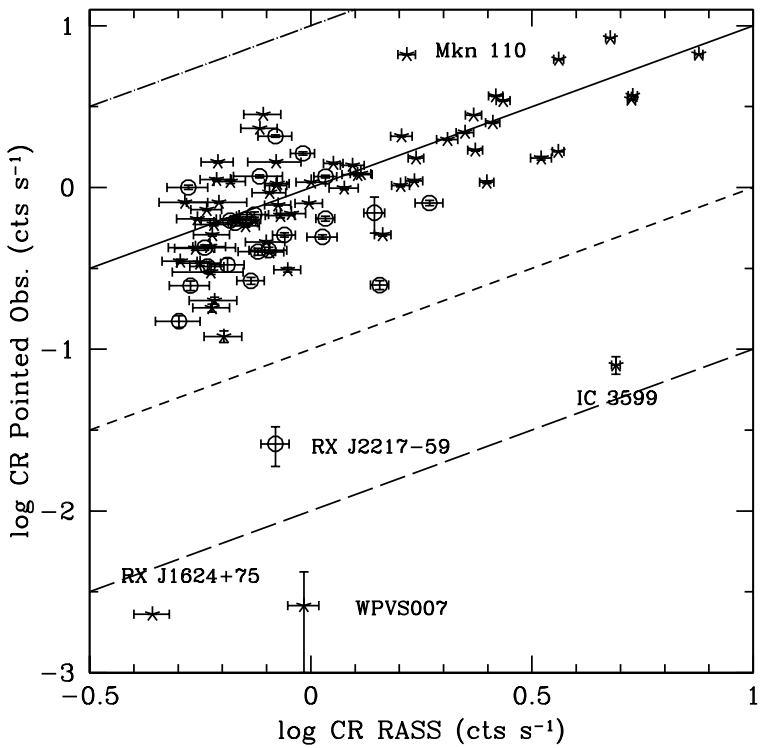

Figure 1. ROSAT All-Sky Survey vs. pointed PSPC observations taken from [17]. The solid line marks constant flux, the short dashed line indicates a drop between RASS and pointed ROSAT observation by a factor of 10 and the long-dashed line marks a drop by a factor of 100 .

\section{HIGH X-RAY VARIABILITY AGN}

\subsection{The NLS1 WPVS 007}

Figure 2 shows the remarkable long-term light curve of WPVS 007. During the RASS, WPVS 007 appeared as an X-ray bright AGN. However, in all follow-up ROSAT observations its X-ray flux dropped by more than a factor of 400 [15]. From the sample presented by [15], WPVS 007 was the AGN with the softest X-ray spectrum. No $\mathrm{X}$-ray photons with energy above $0.5 \mathrm{keV}$ were detected 


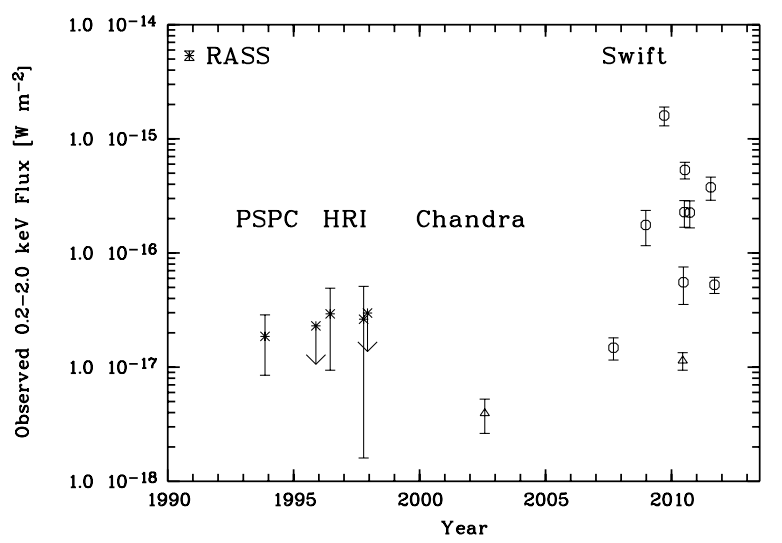

Figure 2. The 0.2-2.0 keV light curve of WPVS 007 [26]. Note that typically Swift does not detect WPVS 007 even in a $5 \mathrm{ks}$ observation. Only significant detections are shown in this plot. The detection limit of the Swift XRT for a $5 \mathrm{ks}$ observation is of the order of $4 \times 10^{-17} \mathrm{~W} \mathrm{~m}^{-2}$. ROSAT, Chandra and Swift data points are marked as stars, triangles, and open circles, respectively.

from WPVS 007. In recent years we have detected WPVS 007 several times. On one occasion, its flux was just a factor of 3 lower than it was during the RASS! What is causing the dramatic X-ray variability in this NLS1?

The X-ray spectra of AGN in X-ray weak states are known to appear complex (e.g. [11]). The X-ray spectra of WPVS 007 during its low state, as seen by Swift, looked completely different than the AGN did in a highstate. The merging of all the Swift XRT data obtained during our intense monitoring campaign of WPVS 007 in September 2011 [26] is shown in Figure 3. X-rays are detected above $5 \mathrm{keV}$ and the spectrum displays strong curvature that can be explained with a partial covering absorber model. Note, however, that such a curvature in the X-ray spectrum in general can also be caused by X-ray reflection (e.g. $[6,11])$. What clarified the behavior seen in WPVS 007 comes from the UV observations. In November 2003 the UV observatory FUSE observed WPVS 007 and detected very deep UV absorption troughs [30]. These strong Broad Absorption Line (BAL) troughs developed within less than a decade. Although variable BALs have been observed before (e.g. [9] and [8]) what makes WPVS 007 so unusual is that the presence of BALs is somewhat unexpected in low-redshift, low-luminosity AGN. BAL troughs are primarily detected in high-luminosity quasars [29]. Nevertheless, [3] and [1] have suggested that BAL QSOs and NLS1s are very similar objects with regards to their high accretion rates. Their black hole masses however, differ significantly with NLS1s being at the low end of the mass distribution and BAL QSOs at the higher end. It has also been found that the rest-frame optical spectra of BAL QSOs look very much alike low-redshift NLS1s (e.g. [32] and [5]). The NLS1 WPVS 007 could be the link between BAL QSOs and NLS1s.

\subsection{The NLS1 Mkn 335}

The NLS1 Mkn 335 is recognized as a consistently X-ray bright AGN, and even one of the 339 X-ray

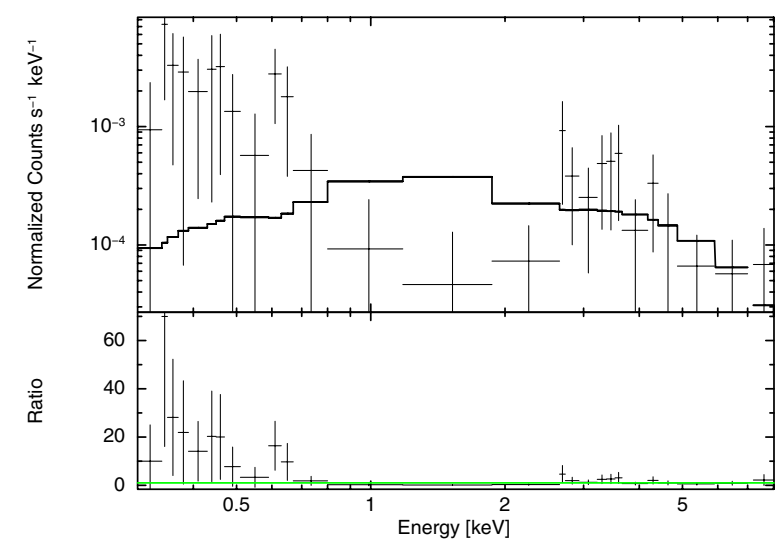

Figure 3. The mean X-ray spectrum of WPVS 007 compiled by merging all significant detections during the 2011 September campaign [26]. The spectrum shown here was binned with 2 counts per bin and fitted by a single power law model with an energy spectral slope $\alpha_{\mathrm{x}}=-0.40$, which does not only result in an unacceptable fit, but is not physical either. The spectrum can be well-fitted by a partial covering absorber model with $N_{\mathrm{H}}=9 \times 10^{22} \mathrm{~cm}^{-2}$ and a covering fraction $f_{\mathrm{pc}}=0.95 \mathrm{using}$ Cash statistics.

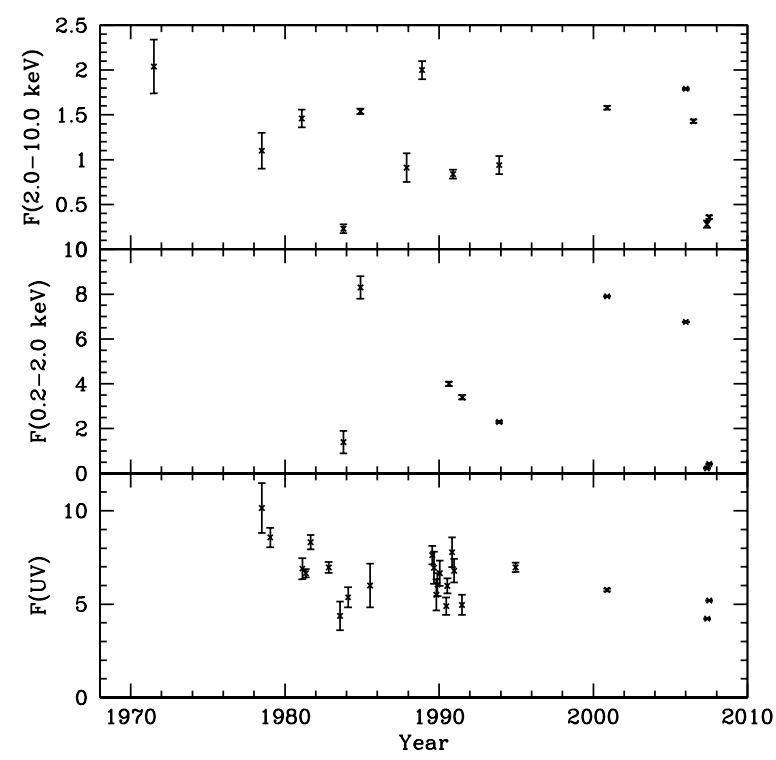

Figure 4. The long-term light curves of Mkn 335 [22] in the $2-10 \mathrm{keV}$ and $0.2-2.0 \mathrm{keV}$ bands (upper and middle panels), and in the UV (lower panel).

sources detected by UHURU [37]. Therefore, it came as a surprise when Swift discovered Mkn 335 in May 2007 in an extremely low X-ray flux state. Figure 4 displays the long-term light curves of Mkn 335 in the 2-10 $\mathrm{keV}, 0.2-2.0 \mathrm{keV}$ and UV bands. After the discovery of Mkn 335 in this unusual state, we initiated a 20ks Target of Opportunity (ToO) observation with XMM. The X-ray spectrum (Figure 5) again shows the typical curvature associated with a partial covering absorber or by blurred reflection of an X-ray source on the disk [22]. Since 2007, we have continued monitoring Mkn 335 with Swift and it has persistently shown dramatic variability in the X-ray and UV as shown in Figure 6. XMM re-observed Mkn 335 in June 2009 for $200 \mathrm{ks}$ and found it in an intermediate 


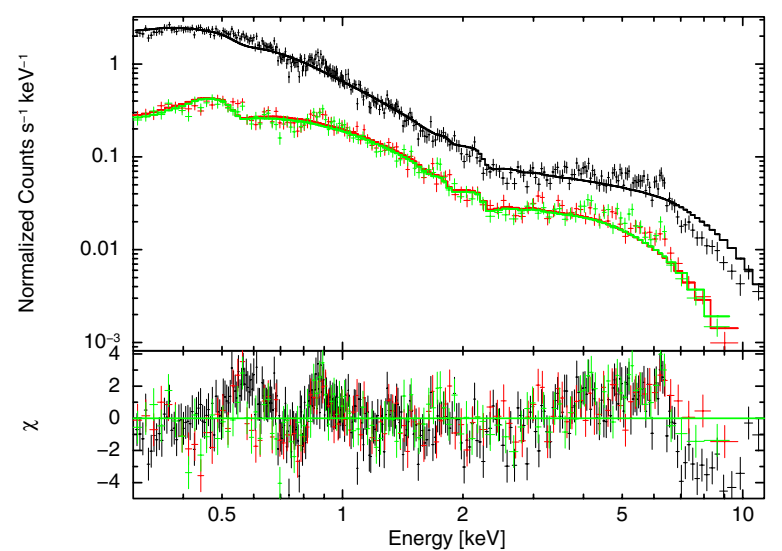

Figure 5. The X-ray spectrum of Mkn 335 during the 20ks 2007 XMM observation when it was in a low-flux state [22]. The residuals in the lower panel compare the data to an absorbed power law model.

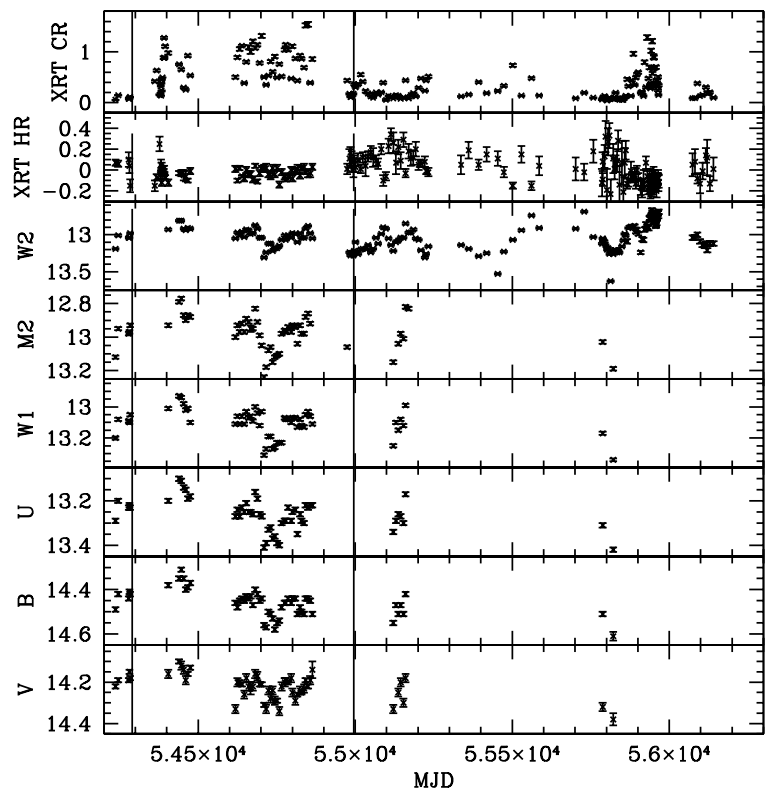

Figure 6. Swift XRT and UVOT light curves of Mkn 335 [25] starting on 2007 May 17. The vertical lines in the plot mark the times of the XMM-Newton observations of Mkn 335 in July 2007 and June 2010.

state. The X-ray data can be interpreted again by a partial covering absorber model [25] or by blurred reflection [13]. It is interesting to note that in the low states, Mkn 335 exhibited strong soft X-ray emission lines in the RGS ([22] and [31]). Mkn 335 is also one of the most variable AGN in the UV (Figure 4). This is very unusual for a NLS1, which generally do not show strong UV variability [24].

\subsection{Other highly variable AGN}

A significant number of highly variable AGN (factors of variability of 20-30) have been detected with various X-ray observatories, including well-known nearby AGN like NGC 4051, NGC 3227, NGC 3786 and NGC 3516. More recently, as already mentioned above, another AGN that has gone through dramatic changes in its X-ray properties is the NLS1 PHL 1092 [34]. This AGN was observed in 2003 with an X-ray luminosity of about $10^{45} \mathrm{erg} \mathrm{s}^{-1}$ [10]. Since then its flux dropped by a factor of 260. However, unlike WPVS 007, this AGN does not show strong UV absorption troughs (see also G. Miniutti's article in these proceedings for details). As mentioned in the introduction, another NLS1 that has shown a dramatic drop in its X-ray flux was RX J2217.9-5941 [18]. Chandra observations of this NLS1 in 2003 February and August confirmed the low X-ray flux state and showed that the Xray spectra exhibit the typical curved shape characteristic of a partial covering absorber [19]. In additions with Swift we are able to identify two more AGN which went into deep X-ray minimum flux states, PG 0844+349 [12] and 1H 0707-495 [7]. Both AGN were observed by XMMNewton in triggered ToO observations after these AGN were discovered in their deep minimum X-ray flux states. The X-ray spectra derived from these XMM observations were discussed in the light of the reflection interpretation by [12] and [7], respectively.

Finally, we discuss the case of IC 3599 ([2] and [14]). Not only has this AGN shown a dramatic drop in its X-ray flux between the RASS and later pointed ROSAT observations, but its optical spectrum also went through dramatic changes. The optical spectrum taken half a year after the X-ray outburst published by [2] showed strong broad $\mathrm{H}, \mathrm{He}$, and coronal Fe lines. When the AGN was observed in later years the $\mathrm{H}$ and $\mathrm{He}$ lines appeared to be normal for a Seyfert galaxy and the coronal $\mathrm{Fe}$ lines continued to fade $[14,27]$. [Note, due to the very unusual optical spectrum after the outburst, IC 3599 was mistakenly classified as a NLS1. However, it never showed one of the characteristics of a NLS1: strong optical Fe II emission.] Several scenarios were mentioned to explain the unusual X-ray outburst of IC3599, including a change in accretion rate in this AGN, perhaps due to a disk instability, or else a tidal disruption event.

\section{THE ORIGIN OF HIGH-AMPLITUDE VARIABILITY IN AGN}

All the sources mentioned above (except IC 3599) look markedly different from TDEs, in every single aspect. In particular, their optical spectra show the classical AGN signatures in the form of permanent, bright, narrow and broad emission lines. Furthermore, their low-state X-ray emission is still bright, and above the contribution from the host galaxy, and their long-term light curves are completely different than the TDE long-term light curves (e.g., [28]). However, highly variable AGN could be a source of 'false alarm' when routinely searching for TDEs in future large-scale surveys, if no follow-up observations are carried out.

One thing that the AGN discussed in Section 2 have in common (except IC 3599) is that they are all NLS1s. These are typically characterized by high Eddington ratios $L / L_{\text {Edd }}$. Often, sources with high $L / L_{\text {Edd }}$ show strong outflows and winds, which can cause an absorber to move in and out of the line of sight. A partial covering absorber can therefore explain at least some of the dramatic flux changes that have been observed in these AGN. 
On the other hand, NLS1 are also characterized by rapid variability and a strong soft excess in the X-rays, that is commensurate with relativistically blurred reflection from the accretion disc (e.g. [4,36]) and light bending [33]. For example, when invoking light bending the extreme variability seen in PHL 1092 requires only very modest changes in the primary emission [34]. Likewise, the very low-flux state in $1 \mathrm{H} 0707-495$ can be described by the very compact nature of the primary X-ray source [7]. While in some cases, the blurred reflection scenario seems to simultaneously provide a more complete description of the rapid (i.e. hourly) variability, along with the extreme longterm changes (e.g. [12]).

Part of this research was based on observations obtained with XMM-Newton, an ESA science mission with instruments and contributions directly funded by ESA Member States and NASA. Swift at PSU is supported by NASA contract NAS500136. This research was also supported by NASA contracts NNX07AH67G, NNX10AK85G, NNX10AF49G, NNX08AT25G, NNX09AP50G, and NNX09AN12G (D.G.).

\section{References}

[1] Boroson, T.A., 2002, ApJ, 565, 78

[2] Brandt, W.N., Pounds, K.A., \& Fink, H.H., 1995, MNRAS, 273, L47

[3] Brandt, W.N., \& Gallagher, S.C., 2000, New Astronomy Review, 44, 461

[4] Crummy, J., et al., 2006, MNRAS, 365, 1067

[5] Dietrich, M., et al., 2009, ApJ, 696, 1998

[6] Fabian, A.C., et al., 1989, MNRAS, 238, 729

[7] Fabian, A.C., et al., 2012, MNRAS, 419, 116

[8] Filiz Ak, N., et al., 2012, ApJ, accepted, arXiv:1208.0836v1

[9] Hamann, F., et al., 2008, MNRAS, 391, L39
[10] Gallo, L.C. et al., 2004, MNRAS, 352, 744

[11] Gallo, L.C., 2006, MNRAS, 368, 479

[12] Gallo, L.C., et al., 2011, MNRAS, 412, 161

[13] Gallo, L.C., et al., MNRAS, submitted

[14] Grupe, D., et al., 1995, A\&A, 299, L5

[15] Grupe, D., et al., 1995, A\&A, 300, L21

[16] Grupe, D., Thomas, H.-C., \& Leighly, K.M., 1999, A\&A, 350, L31

[17] Grupe, D., Thomas, H.C., \& Beuermann, K., 2001, A\&A, 367, 470

[18] Grupe, D., Thomas, H.-C., \& Leighly, K.M., 2001, A\&A, 369, 450

[19] Grupe, D., et al., 2004, AJ, 128, 1524

[20] Grupe, D., et al., 2007a, AJ, 133, 1988

[21] Grupe, D., Komossa, S., \& Gallo, L.C., 2007b, ApJ, 668, L111

[22] Grupe, D., et al., 2008a, ApJ, 681, 982

[23] Grupe, D., Leighly, K.M., \& Komossa, S., 2008b, AJ, 136, 2343

[24] Grupe, D., et al., 2010, ApJS, 187, 64

[25] Grupe, D., et al., 2012, ApJS, 199, 28

[26] Grupe, D., et al., 2012, AJ, in prep

[27] Komossa, S., \& Bade, N., 1999, A\&A, 343, 755

[28] Komossa, S., 2002, in: Reviews in Modern Astronomy 15, 27

[29] Laor, A., \& Brandt, W.N., 2002, ApJ, 569, 641

[30] Leighly, K.M., et al., 2009, ApJ, 701, 176

[31] Longinotti, A.L., et al., 2012, ApJ, in prep.

[32] Marziani, P., et al., 2009, A\&A, 495, 83

[33] Miniutti, G., Fabian, A. C., 2004, MNRAS, 349, 1435

[34] Miniutti, G., et al., MNRAS, in press, arXiv:1207.0694v1

[35] Miniutti, G., et al., 2009, MNRAS, 396, 85

[36] Ross, R.R., Fabian, A. C., 2005, MNRAS, 358 211

[37] Tananbaum, H., et al., 1978, ApJ, 223, 74 